\title{
The SIR dynamic model of infectious disease transmission and its analogy with chemical kinetics
}

\author{
Cory M. Simon ${ }^{1}$ \\ ${ }^{1}$ School of Chemical, Biological, and Environmental Engineering. Oregon State \\ University. \\ Cory.Simon@oregonstate.edu
}

June 17, 2020

\begin{abstract}
Mathematical models of the dynamics of infectious disease transmission are used to forecast epidemics and assess mitigation strategies. We reveal that the classic Susceptible-InfectiousRecovered (SIR) epidemic model resembles a dynamic model of a batch reactor carrying out an autocatalytic reaction with catalyst deactivation. This analogy between disease transmission and chemical reactions enables the cross-pollination of ideas between epidemic and chemical kinetic modeling.
\end{abstract}

\section{1 introduction}

Mathematical models of the dynamics of infectious disease transmission [1. 2] are useful for forecasting epidemics, assessing intervention strategies, and inferring properties of diseases.

In compartmental epidemic models [3], each member of the population is categorized based on their disease status in addition to, possibly, their attributes and the treatment they received. The dynamics of disease transmission are then typically modeled with differential equations that describe the flow of individuals to and from the compartments as the population mixes, the disease spreads, and infected individuals progress through the stages of the disease. Differential equations are a natural choice because we can make reasonable assumptions about the rates at which people acquire the infection and progress through the stages of the disease.

In this article, we highlight the analogy between the dynamics of disease transmission and chemical reaction kinetics while formulating and analyzing the classic SIR compartmental epidemic model [4. 5]. 


\section{2 the SIR model}

In the classic SIR model of an epidemic [2 4 4, 6 , 7], each member of the population belongs to one of three compartments: Susceptible, Infectious, or Recovered.

\section{1 the reactions}

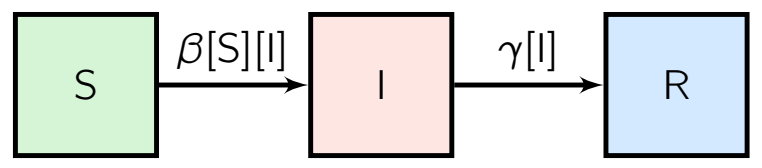

Figure 1: The SIR model. The boxes represent the set of Susceptible, Infectious, and Recovered individuals. The arrows represent flow from one compartment to another and are annotated with per capita flow rates.
Susceptible folks can contract the disease if they come into contact with an infectious individual. Once infected, they move into the infectious compartment, assuming zero delay between infection and the ability to transmit the disease. This is analogous to an irreversible autocatalytic chemical reaction [8] between a reactant, $\mathrm{S}$, and catalyst, I:

$$
\mathrm{S}+\mathrm{I} \longrightarrow 2 \mathrm{I}
$$

Infectious individuals eventually recover or die from the disease, entering the recovered compartment, and then cannot transmit the disease or contract it again. This is analogous to a reaction where the catalyst, I, irreversibly degrades or converts to a deactivated form, R:

$$
\mathrm{I} \longrightarrow \mathrm{R}
$$

The R category is sometimes called the Removed category [10] instead, emphasizing its inclusion of disease-induced deaths.

We assume that recovery from the disease confers permanent immunity to reinfection, thus neglecting the possibility of an $\mathrm{R} \longrightarrow \mathrm{S}$ reaction.

So, the SIR model of an epidemic is analogous to an autocatalytic reaction ( $r \times n$. \{1\}) with catalyst deactivation (rxn. \{2\}). An infectious individual (the catalyst, l), (i) upon contacting (colliding with) a susceptible individual (the reactant, S), can convert them into another infectious individual (another catalyst particle) and (ii) recovers (deactivates) with time. Fig. 1 depicts the flow of individuals through compartments under the SIR model, induced by rxns. \{1 $\}$ and $\{2\}$.

\section{2 the dynamic mathematical model}

Mathematically, the SIR model [4.6711] is equivalent to a dynamic model of a well-mixed, isothermal batch reactor carrying out the two homogeneous, elementary rxns. \{1] and \{2\}.

As in a (closed) batch reactor, we neglect immigration and emigration (hence the absence of flow to/from external populations in Fig.11. Moreover, we take births and deaths (from causes other than the disease) to be negligible over the (fast) time scale of the epidemic.

Let $[S](t),[I](t)$, and $[R](t)$ be the fraction of the population that is susceptible, infectious, and recovered, respectively, at time $t$. Considering a large population, we treat $[S],[I]$, and $[R]$ as continuous variables. 
the incidence rate. Assuming their spatial mixing is uniform [12, 13], we invoke the law of mass action to model the rate at which susceptible and infectious individuals "react" via bimolecular, autocatalytic rxn. \{1]. The incidence rate of the disease, i.e. the number of new infections per unit time [11], is then $\beta[S][I]$ (per capita). A symmetric, bilinear function of $[S]$ and $[I]$, intuitively, the incidence rate doubles if [I] ([S]) doubles while $[S]$ ([I]) is fixed. The second-order transmission rate constant $\beta>0$ is the product of the average frequency of contacts of a person and the transmissibility of the disease (the probability of transmission conditioned upon contact).

the recovery rate. We model the rate at which infectious individuals "deactivate" (recover) via rxn. \{2\} with first-order kinetics, i.e., as $\gamma[I]$ (per capita). The inverse of the first-order recovery rate constant $\gamma>0$ is the average time period that an infected individual is infectious [14].

With the assumptions above (see Fig.1 1 for flows), we arrive at the following set of nonlinear, coupled differential equations that comprise the SIR dynamic model of infectious disease transmission:

$$
\begin{aligned}
& \frac{d[\mathrm{~S}]}{d t}=-\beta[\mathrm{S}][\mathrm{I}] \\
& \frac{d[\mathrm{I}]}{d t}=\beta[\mathrm{S}][\mathrm{I}]-\gamma[\mathrm{I}] \\
& \frac{d[\mathrm{R}]}{d t}=\gamma[\mathrm{l}] .
\end{aligned}
$$

The only two parameters in the SIR model are the transmission and recovery rate constants, $\beta$ and $\gamma$, respectively. While the average duration of infectiousness, $\gamma^{-1}$, can be estimated from contact tracing or shedding studies [15], $\beta$ could be identified by fitting differential eqns. 1 13 to epidemic time series data (case counts) [11, 13], much like identifying a reaction rate constant from concentration time series [16].

Addition of eqns. 11 3 confirms the population is closed and demography is neglected, i.e., [S] $(t)+$ $[\mathrm{I}](t)+[\mathrm{R}](t)=1, \forall t \geq 0$. As a consequence, eqns. 1 1and 2 fully determine the SIR model dynamics, with $[R](t)=1-[S](t)-[I](t)$.

\section{3 the replacement and basic reproduction numbers}

Two important numbers aid our characterization and understanding of SIR model dynamics: the replacement number, $r$, and the basic reproduction number, $\mathcal{R}_{0}$.

The replacement number, $r=r(t)$, is the expected number of folks (directly) infected by a typical infectious individual, mixing in the population, over the course of their infectiousness [2]. Because the concentration of susceptible folks $[S]=[S](t)$ influences the frequency that a typical infectious individual contacts a susceptible individual, $r$ changes over time. In the SIR model, a typical infectious individual is expected to be infectious for a time period of $\gamma^{-1}$ and, during this time, produce $\beta[S](t)$ new infections per unit time (incidence rate per infectious individual). The replacement number is therefore:

$$
r=r(t)=\frac{\beta}{\gamma}[S](t)
$$


The basic reproduction number, $\mathcal{R}_{0}$, is defined as the initial replacement number when one infectious individual is introduced into an all-susceptible population [2 7]. Because the entire population is susceptible in this context, $\mathcal{R}_{0}$ in the SIR model is the replacement number in eqn. 4 when [S] $\approx 1$ :

$$
\mathcal{R}_{0}=\frac{\beta}{\gamma}
$$

i.e., $\mathcal{R}_{0}$ is the expected number of infections directly caused by a single infectious individual introduced into an entirely susceptible population over the course of their infectiousness [2].

The replacement number, $r$, and basic reproduction number, $\mathcal{R}_{0}$, are both dimensionless and are properties of both the disease and the population [17]. While $r=r(t)$ changes with time, $\mathcal{R}_{0}$ is constant and defined only at the initial stage when one infectious individual is introduced to an allsusceptible population. Notably, the two numbers are related via $r(t)=\mathcal{R}_{0}[S](t)$.

If the basic reproduction number $\mathcal{R}_{0}$ is large (small), the infected are infectious for a long (short) period of time, the disease is (not) easily transmitted, and/or the average frequency of contacts is high (low). Under the analogy with chemical kinetics, since the activity and longevity of the catalyst, I, are embedded in $\beta$ and $\gamma$, respectively: $\mathcal{R}_{0}$ is large (small) if the catalyst has a high (low) activity and/or remains active for a long (short) time. Because $r=\mathcal{R}_{0}[\mathrm{~S}]$, these remarks also hold for the replacement number, $r$. Though, $r$ decreases as the concentration of the reactant, [S], decreases, owing to the reduced frequency that any given catalyst particle encounters a reactant particle to catalyze its conversion into another catalyst particle by rxn. \{1\}.

\section{SIR model dynamics}

In the SIR model, what happens if we introduce a small number of infectious individuals into a large population of susceptible individuals? This is akin to injecting our deactivating auto-catalyst, I, into a well-mixed batch reactor containing pure $\mathrm{S}$. The corresponding initial conditions are:

$$
\begin{aligned}
{[S](0) } & =[S]_{0} \\
{[\mathrm{I}](0) } & =[\mathrm{I}]_{0} \\
{[\mathrm{R}](0) } & =0,
\end{aligned}
$$

with $[S]_{0}+[I]_{0}=1,[S]_{0},[I]_{0}>0$, and $[I]_{0}<<1$. We consider $[R](0)=0$ for the interesting case where a population is exposed to a novel pathogen to which it has no immunity.

\section{1 the replacement number determines $[I]^{\prime}(t)$}

The replacement number $r(t)$ in eqn. 4 is key to understanding SIR model dynamics. By inspection of eqn. 2 [ $[1](t)$ is increasing at time $t$ if the replacement number $r(t)>1$ and decreasing if $r(t)<1$. This is intuitive: if the typical infectious person mixing in the population is expected to infect less than one susceptible person before they recover, they are not expected to replace themselves with a new infectious individual to propagate the disease, and [I] $(t)$ decreases. Note $r(t)<1 \Longleftrightarrow$ $[\mathrm{S}](t)<\mathcal{R}_{0}^{-1}$. 


\section{2 does an epidemic ensue?}

We first address a qualitative question: given the initial conditions in eqn. 6,8, does the disease invade the population? The outcome depends on the initial replacement number $r_{0}:=r(0)=$ $\mathcal{R}_{0}[\mathrm{~S}]_{0}$ in a threshold manner. If $r_{0}>1,[\mathrm{I}](t)$ initially increases. i.e., the disease spreads; an epidemic ensues. If $r_{0}<1,[\mathrm{I}](t)$ initially decreases and, since $[S](t)$ is a decreasing function of time (see eqn. 1), decays to zero. i.e., the disease dies out; an epidemic does not ensue.

Under the chemical reaction analogy, if $r_{0}<1\left(r_{0}>1\right)$, the injected catalyst particles deactivate via rxn. \{2\} faster (slower) than they catalyze rxn. \{1\} to convert the reactant, $S$, into more catalyst, I, to propagate autocatalytic rxn. \{1\}.

For our remaining analysis, we take $r_{0}>1$ and further analyze the dynamics of an SIR epidemic.

\section{3 initial exponential growth}

Early in the epidemic, the number of infectious folks grows, approximately, exponentially with growth rate $\left(r_{0}-1\right) \gamma$ :

$$
[I](t) \approx[I]_{0} e^{\left(r_{0}-1\right) \gamma t}
$$

This follows from eqn. 2 if we approximate $[S](t) \approx[S]_{0}$, valid only in the initial stage of the epidemic; as the disease spreads, [S] decreases and diminishes the replacement number. Eqn. 9 is thus an overestimate.

Since eqn. 9 is also a valid initial approximation for $r_{0}<1$, it reinforces that an epidemic will not ensue if $r_{0}<1$, since $[I](t)$ would then decay approximately exponentially.

\section{4 a simulation of an SIR model epidemic}

Fig. 2 shows the numerical solution to eqns. 13 with initial conditions in eqns. 6.8 for $\mathcal{R}_{0}=2$ and $[I]_{0}=10^{-5}$ (see Sec. A1.1 for code). Initially, the concentration of infectious folks, [I], grows approximately exponentially (see Fig. A8 for a comparison of $[I](t)$ with eqn. 9). The concentration of susceptible folks, [S], decreases monotonically as the disease invades. As a result, the frequency with which any given infectious individual comes into contact with a susceptible individual decreases. In conjunction with the infectious folks recovering, this eventually causes the (net) growth rate of [I] to diminish and, when the replacement number $r$ in eqn. 4 drops below

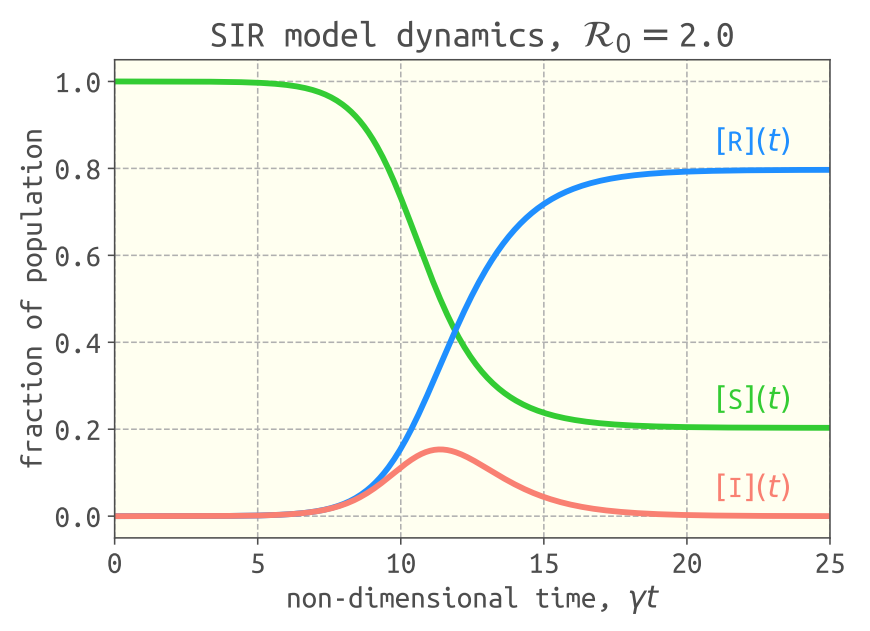

Figure 2: Numerical approximation of the solution to the SIR model in eqns. 1 $3\left(\mathcal{R}_{0}=2\right)$ with initial conditions in eqns 6 8 $\left([I]_{0}=10^{-5}\right)$. 
one, causes [I] to decay. The epidemic self-

extinguishes, i.e., $\lim _{t \rightarrow \infty}[I](t)=0$ [18]. The $R$ category accumulates the folks that have been infected by and have recovered from the disease, $[R](t)=\gamma \int_{0}^{t}[I](\tau) d \tau$ (see eqn. 3 with initial condition 8. Notably, the disease does not infect the entire population, even after an infinite amount of time. i.e., the epidemic self-extinguishes not because the population is depleted of susceptible folks, but rather because it is depleted of infectious folks [6 [3].

Under the chemical kinetics analogy, the autocatalytic rxn. (1] begins to slow down after the concentration of the reactant, [S], decreases sufficiently. Once the replacement number $r(t)$ drops below one (i.e. once [S] drops below $\mathcal{R}_{0}^{-1}$ ), the catalyst, I, is deactivating via rxn. $\{2\}$ faster than it is converting the reactant, $\mathrm{S}$, into more catalyst to replenish itself via rxn. \{1\}. Consequently, the catalyst concentration, [l], begins to drop and decays to zero. Owing to catalyst deactivation ( $r \times n$. 22\}), not all reactant, $\mathrm{S}$, is consumed, even after an infinite amount of time.

\section{5 solution in the ([S], [I]) phase space}

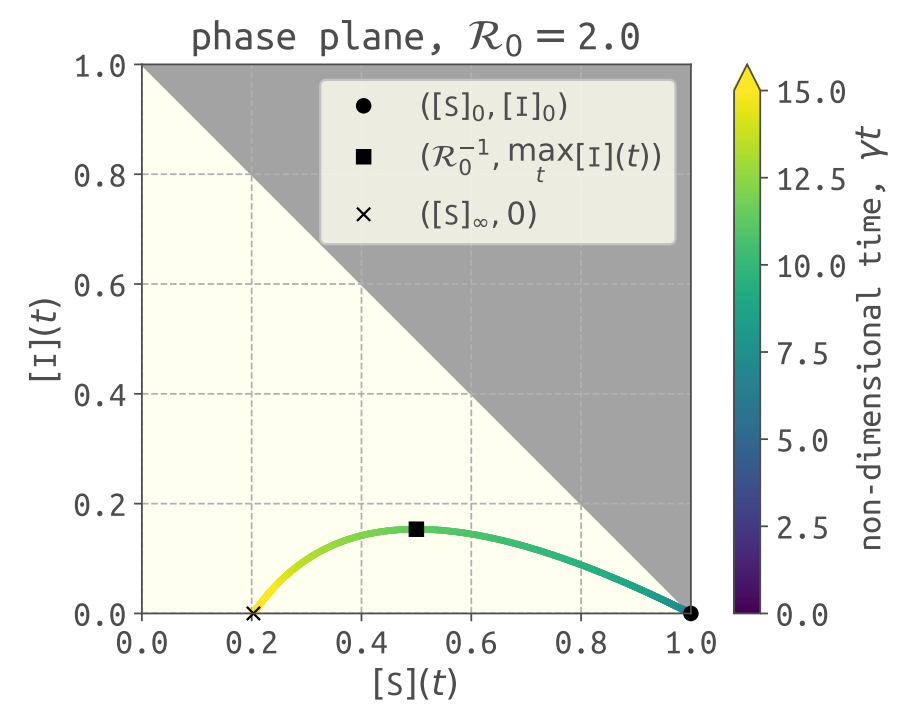

Figure 3: The trajectory of the solution to the SIR model $\left(\mathcal{R}_{0}=2\right.$, initial conditions in eqns 6 6 with $[1]_{0}=10^{-5}$ ) in the ([S], [I]) phase plane, given by eqn. 10. The trajectory is colored according to time. The initial conditions, peak prevalence of infectious folks, and final conditions are marked. Since $[S](t)+[I](t) \leq 1$, solutions in the gray region are infeasible.
We can analytically find the trajectory of the solution to eqns. 122 in the ([S], [I]) phase plane. Dividing eqn. 2 by eqn. 17takes us into the phase plane by giving a differential equation with a $\frac{d[1]}{d[\mathrm{~S}]}$ derivative, with the view of [I] as a function of [S]. Separating, integrating, and applying the initial conditions in eqns. 6 and 7 we arrive at the solution path [3]:

$$
[\mathrm{I}](t)=1-[\mathrm{S}](t)+\frac{1}{\mathcal{R}_{0}} \log \left(\frac{[\mathrm{S}](t)}{[\mathrm{S}]_{0}}\right) .
$$

Fig. 3 shows the trajectory given in eqn. 10 $\left(\mathcal{R}_{0}=2,[1]_{0}=10^{-5}\right)$ and reinforces that $[S](t)$ decreases monotonically with time, that $[I](t)$ increases, peaks, then diminishes to zero, and that a fraction of the population remains susceptible after the epidemic dies out.

\section{6 final size}

Given the epidemic (autocatalytic reaction) dies out before all of the susceptible folks (reactant) have been infected, what fraction of the population (reactant) remains susceptible (unreacted) after the epidemic ends? 


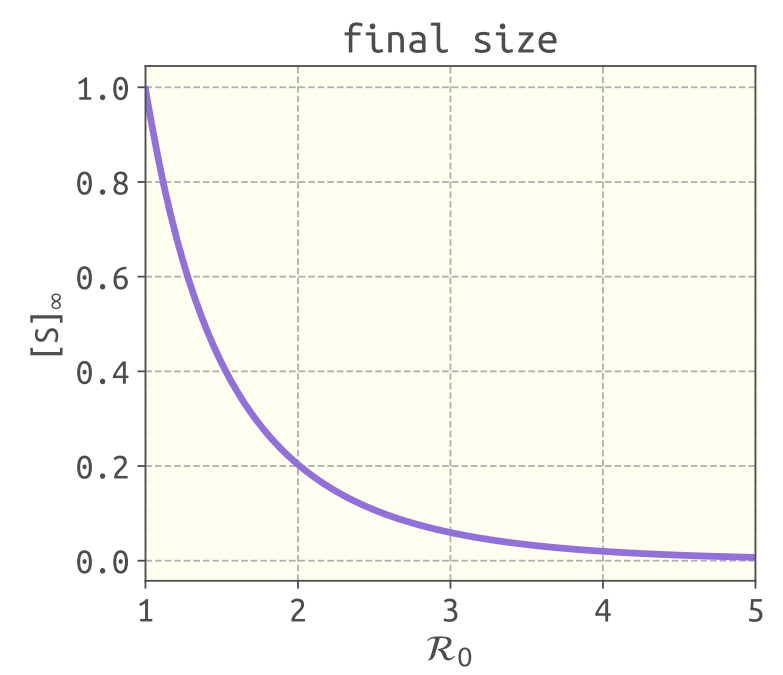

(a)

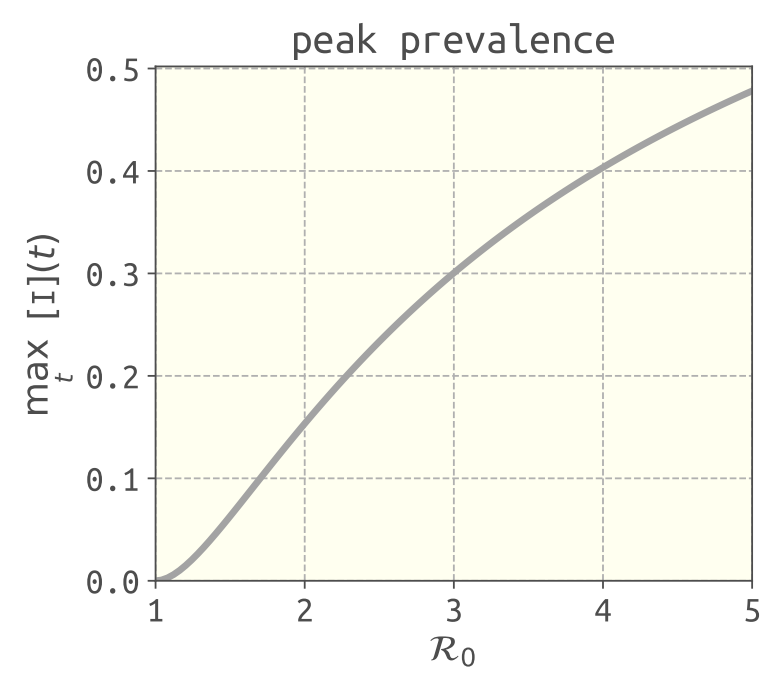

(b)

Figure 4: The fraction of the population that (a) remains susceptible (never infected) after the SIR epidemic runs its course, $[S]_{\infty}$, via eqn. 11 and $(b)$ is infected at peak prevalence, $\max _{t}[\mathrm{l}](t)$, via eqn. 12 both as a function of $\mathcal{R}_{0}$. Corresponds to initial conditions in eqns. 68 with $[1]_{0}=10^{-5}$.

We find an implicit equation for $[S]_{\infty}:=\lim _{t \rightarrow \infty}[S](t)$ by taking the limit $t \rightarrow \infty$ in eqn. 10

$$
0=1-[S]_{\infty}+\frac{1}{\mathcal{R}_{0}} \log \left(\frac{[S]_{\infty}}{[S]_{0}}\right)
$$

We used the fact that the epidemic eventually dies out, $\lim _{t \rightarrow \infty}[I](t)=0$. $[S]_{\infty}$ is the unique root of eqn. 11 in $\left(0, \mathcal{R}_{0}^{-1}\right)[2]$. The fraction of the population infected over the course of the epidemic is $\lim _{t \rightarrow \infty}[R](t)=:[R]_{\infty}=1-[S]_{\infty}$ since the $R$ category accumulates those that have recovered from the disease. Fig. 4 a shows that, as $\mathcal{R}_{0}$ increases from one, more of the population will be infected over the course of the epidemic.

\section{7 peak prevalence of infectious folks}

The peak prevalence of infectious folks, $\max _{t}[l](t)$, is important because it determines the maximum burden on healthcare. Both eqn. 10 and eqn. 2 show that the maximum in $[\mathrm{I}](t)$ (look for $\frac{d[\mathrm{I}]}{d[\mathrm{~S}]}=0$ or $\frac{d[l]}{d t}=0$, respectively) occurs when $[S](t)=\mathcal{R}_{0}^{-1}$ (i.e., when replacement number $r(t)=1$ ). Via eqn. 10 then:

$$
\max _{t}[I](t)=1-\frac{1}{\mathcal{R}_{0}}\left[1+\log \left(\mathcal{R}_{0}[S]_{0}\right)\right]
$$

Fig. $4 \mathrm{~b}$ shows how the peak prevalence of infectious folks increases with $\mathcal{R}_{0}$. Before the maximum, the replacement number $r(t)>1$ and $[I](t)$ is increasing; after the peak, $r(t)<1$ and $[I](t)$ is decreasing to zero. 


\section{8 herd immunity}

A population is conferred herd immunity when a sufficient fraction is immune to the disease so as to indirectly protect it from invasion of the disease upon the introduction of an infectious individual. Notably, such immunity could be acquired by either previous infection or by vaccination. [2]

What fraction $v$ of a population must be immune to achieve herd immunity? [I] $(t)$ will decrease upon introducing an infectious individual if the replacement number $r(t)<1$, i.e. if $[S]<\mathcal{R}_{0}^{-1}$. Thus, a fraction $v>1-\mathcal{R}_{0}^{-1}$ of the population must be immune to achieve herd immunity. Fig. 5 shows the region $v>1-\mathcal{R}_{0}^{-1}$ (gray) and illustrates that, if $\mathcal{R}_{0}$ is large (small), more (less) of the population must be immune to prevent an epidemic via herd immunity.

In the chemical kinetics analogy, herd immunity results from reducing the concentration of the reactant, [S], so that a catalyst particle, I, fed to the reactor is expected to deactivate before it encounters an $\mathrm{S}$ particle and auto-catalyzes rxn. \{1\} to replace itself. i.e., to achieve herd immunity, [S] must be reduced sufficiently to make the replacement number $r$ less than one.

To account for vaccination dynamics in the midst of an epidemic, we can modify the SIR model by including induced flow in Fig. 1 from the $S$ category directly to the R category, representing the administration of a vaccine to susceptible folks that confers complete immunity [11 19. 20].

An interesting comparison is when herd immunity is achieved through a vaccine $(S \longrightarrow R$ ) versus through infection and recovery $(S \longrightarrow$ $\mathrm{I} \longrightarrow \mathrm{R}$ ). One obvious benefit of the path $\mathrm{S} \longrightarrow \mathrm{R}$ over the path $\mathrm{S} \longrightarrow \mathrm{I} \longrightarrow \mathrm{R}$ is avoiding disease-induced suffering and, possibly, death. Anyway, administering an effective vaccine to a fraction $v=1-\mathcal{R}_{0}^{-1}$ of an

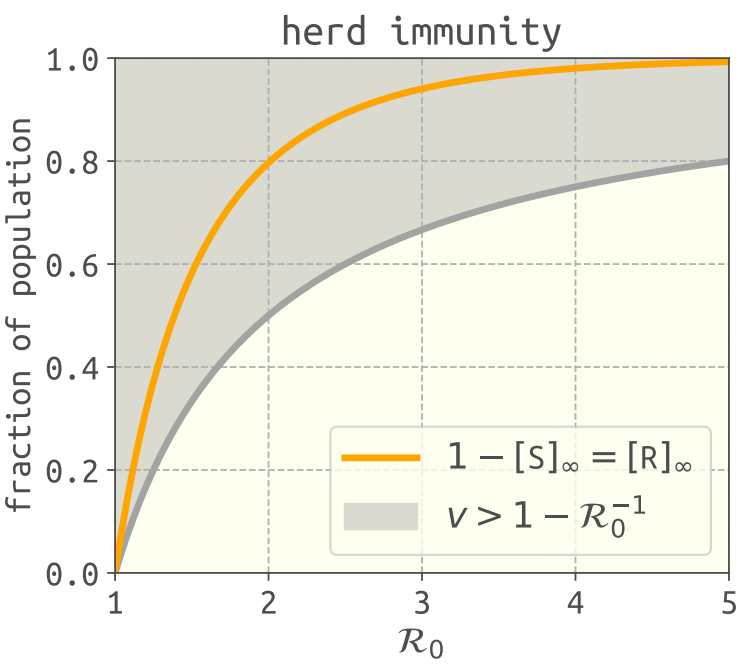

Figure 5: Herd immunity. The gray region shows the fraction $v>1-\mathcal{R}_{0}^{-1}$ of a population that must be immune to achieve herd immunity. Such immunity could be conferred by vaccination or by previous infection. For comparison, the orange line shows $[R]_{\infty}$, the number of folks that will be infected over the course of an SIR epidemic without any vaccination $\left([l]_{0}=10^{-5}\right)$.

all-susceptible population will suffice to achieve herd immunity. However, this does not imply that the same fraction will be infected if herd immunity is instead achieved through infection and recovery, by introducing infectious individuals and allowing the epidemic to run its course. Fig. 5 also shows (orange curve) $[R]_{\infty}$, the fraction of the population that will be infected by the disease over the course of an SIR epidemic without any vaccination. A significantly greater fraction of the population will be infected by the disease if we let it run its course than would have needed vaccination to achieve herd immunity. When [S] $(t)$ reaches $\mathcal{R}_{0}^{-1}$ in an SIR epidemic without vaccination, $[I](t)$ is at its maximum (see eqn. 10 and Fig. 3); these infectious individuals will infect more susceptible folks before they all recover, even though on average each is 
expected to recover before they infect a susceptible individual ( $r<1$ after this point).

\section{9 conclusion: $\mathcal{R}_{0}$ is a useful tool}

The basic reproduction number $\mathcal{R}_{0}$ is a property of an infectious disease within a population. The average frequency of contacts of an individual in the population, the transmissibility of the disease, and the average duration of infectiousness are all embedded in $\mathcal{R}_{0}$. In the SIR model, $\mathcal{R}_{0}$ influences if the disease invades the population, the initial exponential growth rate of infectious folks, how many are infected over the course of the epidemic, the peak prevalence of infectious folks, and how many must be vaccinated to achieve herd immunity.

\section{4 extensions to the SIR model}

The SIR model is a very simple epidemic model, but we can extend it to model other epidemiological factors and prevention/control measures by introducing:

additional compartments. For example, the SEQIJR model [22] introduces three additional compartments: Exposed, Quarantined, and Isolated (J). Usually, the E compartment is included to model the latent period of a disease; exposed individuals have been exposed to and infected by the disease but are not yet infectious [3.23]. The $\mathrm{Q}$ and J compartments are included to model the control measures of quarantining individuals exposed to the disease and isolating infectious individuals, respectively. Members of the $\mathrm{Q}$ and J compartments contact susceptible folks with a reduced frequency [3]. See Fig. 6a. As another example, to distinguish between Asymptomatic and symptomatic infectious individuals [24], which may have different recovery rates, frequencies of contacts, and transmissibilities, we can introduce an A compartment. Finally, to account for different mixing patterns, infectiousness, and susceptibility among different age groups [3], age-structured compartmental models partition the $S$ and I compartments into age groups.

Additional compartments complicate the derivation of the basic reproduction number $\mathcal{R}_{0}$ from the model [25]. Still, the peak prevalence of infectious folks and final size of the epidemic increase with $\mathcal{R}_{0}$ in most models [26]. Notably, a more precise definition of $\mathcal{R}_{0}$ is needed if compartments are introduced to account for heterogeneity in the population [27].

time-varying parameters. A time-varying transmission rate constant $\beta=\beta(t)$ can model changes in the frequency of social contacts within the population or in the transmissibility of the disease. For example, members of the population may (voluntarily or induced by policy) change/adapt their behavior during the epidemic, e.g., by reducing their frequency of social contacts, practicing social distancing, taking hygiene measures, etc. [18, 28 29]. A periodic $\beta(t)$ can model seasonality of an infectious disease [30 31]. A time-varying recovery rate constant $\gamma=\gamma(t)$ can model changes in the average time period of infectiousness, e.g., a reduction of $1 / \gamma$ by administering a drug to infected patients [18]. Reducing the transmission rate constant $\beta$ and/or the average time period of infectiousness $1 / \gamma$ reduces the replacement number $r$ (see eqn. 4); if $r$ is reduced below one, the prevalence of infectious folks will decrease.

births, deaths, and loss of immunity. To model infectious disease transmission over longer time scales, we can modify the closed SIR model to account for births and deaths (caused by factors other 


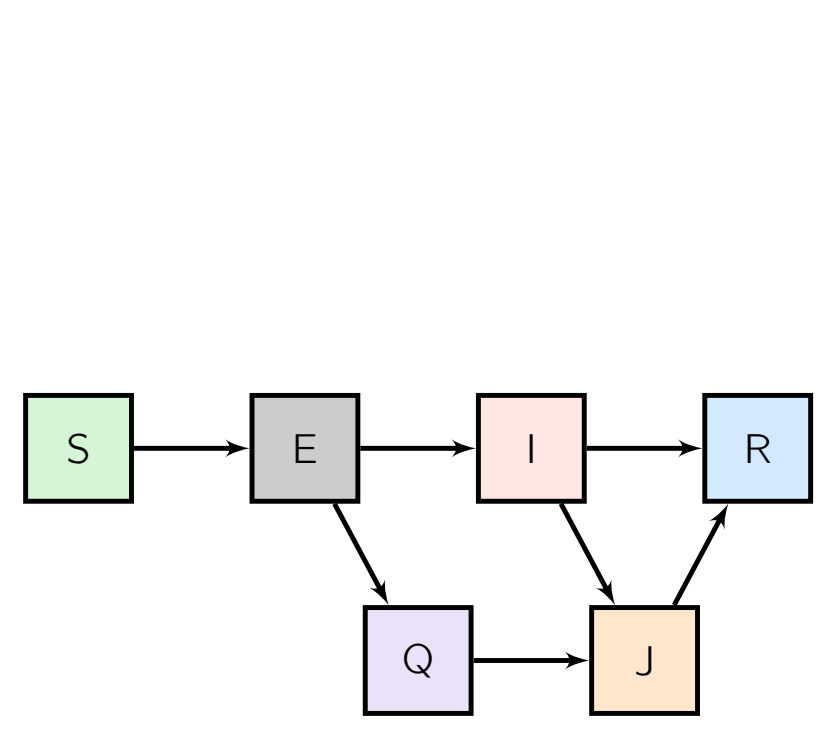

(a)

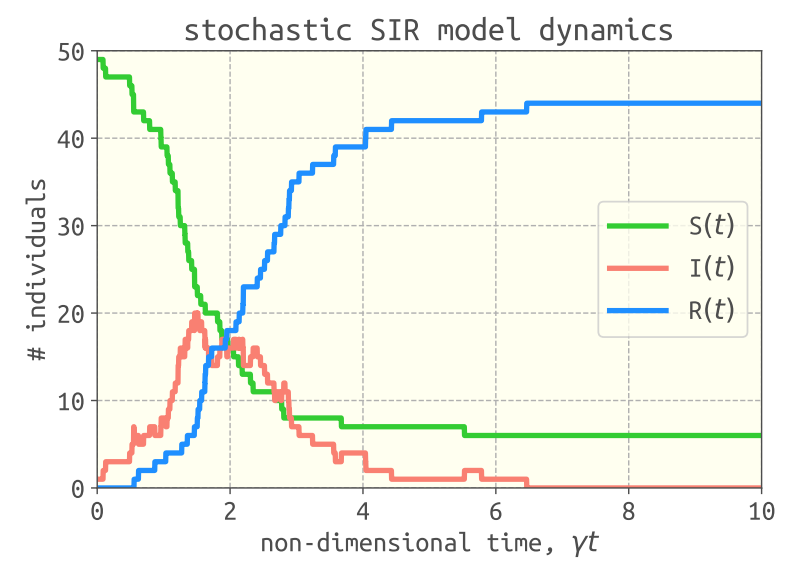

(c)

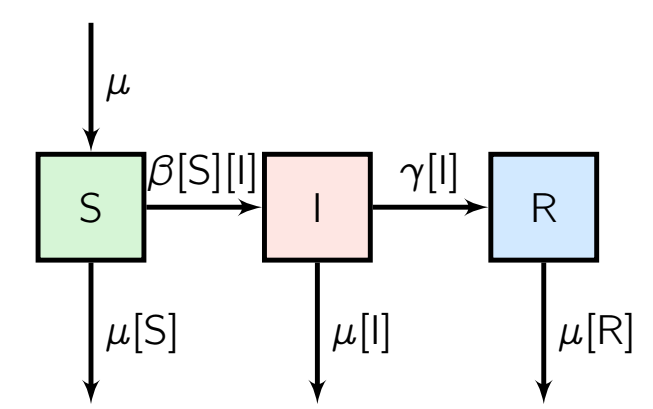

phase plane

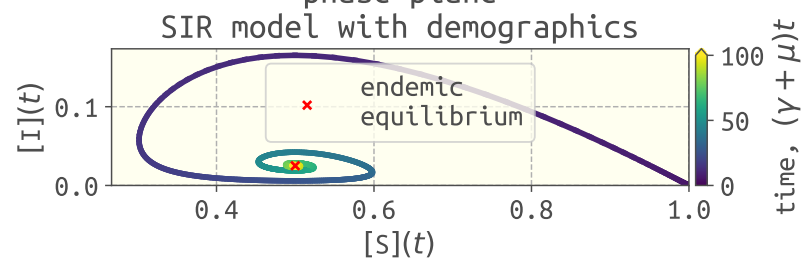

(b)

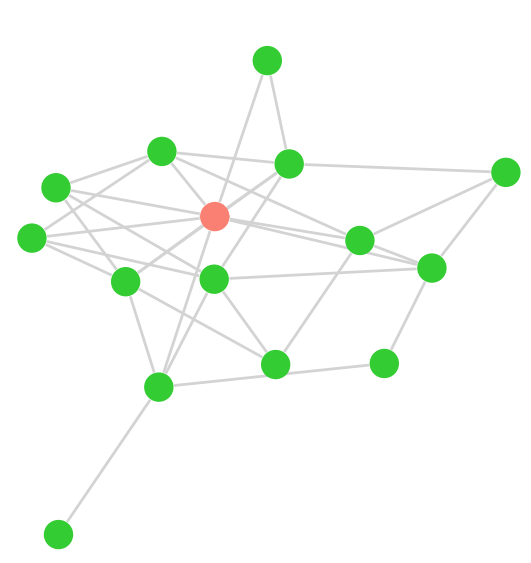

(d)

Figure 6: Extensions to the SIR model. (a) Introducing additional compartments. Exposed individuals have been exposed to and infected by the disease, but cannot yet transmit the disease, due to a latent period. Owing to control measures, quarantined (Q) and isolated (J) individuals contact susceptible folks with a reduced frequency. (b) When demographics (births and natural deaths, with rate constant $\mu$ ) are included in the SIR model, [I] $(t)$ can exhibit damped oscillations that settle on an endemic equilibrium. The phase plane shows the dynamics for $[1]_{0}=1 e-5, \beta /(\mu+\gamma)=2$, and $\mu /(\mu+\gamma)=0.05$ (unrealistically large to clearly see $\lim _{t \rightarrow \infty}[1](t)>0$ ). [2] (c) A simulation of the (stochastic) SIR continuous time Markov chain model [21] for a small population of 50 individuals, with one initially infectious ( $\mathcal{R}_{0}=2$ ). (d) In a contact network model, nodes represent individuals and edges represent contacts. Here, the highly connected infected node (red) can infect its nine susceptible (green) neighbors. 
than the disease) by allowing flow into the $S$ compartment and flow out of all compartments, respectively. In addition, as opposed to assuming recovery from infection confers permanent immunity, we can address temporary immunity and model the loss of immunity by introducing a $\mathrm{R} \longrightarrow$ S reaction. Because births and the loss of immunity continuously add to the susceptible pool, $[I](t)$ in the SIR model with births, deaths, and loss of immunity can exhibit damped oscillations that settle on an endemic equilibrium, where the disease remains in the population indefinitely (i.e., [I] is maintained at a non-zero value). See Fig. 6b. At an endemic equilibrium, the replacement number is maintained at one so that $[I](t)$ is neither increasing nor decreasing. [2, 32, 33]

stochasticity. As opposed to the deterministic differential eqns. 113 we can introduce randomness into the SIR model to account for the stochastic and uncertain nature of human interaction and disease transmission [21 34 35]. Stochastic epidemic models aim to describe the probabilistic distribution of outcomes, e.g., the distribution of $[S]_{\infty}$ [34 35]. Stochasticity can be particularly important for small populations and in the early stage of an epidemic when there are small numbers of infectious individuals [35]. Analogously, stochastic models of chemical reaction dynamics are necessary when the reactants are not abundant, such as in a biological cell [36. 37]. See Fig. 6C for an example of a stochastic simulation of the SIR model.

more realistic probabilistic distributions of the infectious time period. The probabilistic implication of first-order decay in eqn. 3 for a single infected individual is that their time period of infectiousness is an exponentially distributed random variable with mean $\gamma^{-1}$ [3]. More realistic probabilistic distributions of the time period of infectiousness can be built into the SIR model [38 39].

spatial heterogeneity. We can model spatial heterogeneity of an epidemic in a discrete [40] or continuous [11] manner. Modeling the spatial movement of susceptible and infectious individuals in a continuous space as a diffusive process results in reaction-diffusion equations, also familiar to chemists and chemical engineers [11]. Compartmental, metapopulation epidemic models with travel between spatially segregated regions resemble models of multiple batch reactors connected with pipes that allow flow between them [40.41].

more detail/structure in contact patterns. Agent-based and network epidemiological models can account for structure and heterogeneity in contact patterns among members of a population [42-46]. In a contact network model, each individual of the population is represented by a node, and edges between nodes represent interactions that could result in disease transmission if one of the nodes becomes infectious [45 47]. See Fig. 6d. The dynamics of disease transmission on a contact network depend significantly on its structure, e.g., on the degree distribution, node clustering, and correlation between the degrees of connected nodes [45]. Intuitively, the dynamics of an SIR model on large, static, random $k$-regular contact networks (a random network where each node has $k$ neighbors) closely approximate those of eqns. 1. 3 which assume homogeneous mixing [45]. In contrast, the dynamics of SIR models on highly heterogeneous contact networks, such as those with heavy-tailed degree distributions that capture "superspreaders" [48 49], deviate significantly from eqns. 1.3. Directed networks can model asymmetric contacts that can only transmit the disease oneway, such as donated blood transfusions [46, 50].

vectors that transmit the pathogen from host to host. Some infectious diseases are primarily transmitted from one host to another host by living vectors that can acquire and carry the infectious agent. For example, mosquitoes can acquire an infectious agent (e.g., the virus that causes dengue 
fever or the parasite that causes malaria) from feeding on the blood of an infected human, then transmit the infectious agent to another, susceptible human when feeding on their blood. SIR-like models of diseases transmitted by vectors include an incidence term $\beta[S]\left[I_{V}\right]$, where $\left[I_{V}\right]$ is the concentration of infectious vectors and $\beta$ includes the frequency that the vector bites the hosts and the probability of transmission conditioned upon a bite. [51.52]

\section{5 conclusions}

Mathematical models of the dynamics of disease transmission are used to forecast epidemics and assess mitigation strategies. The analogy between disease transmission and an autocatalytic reaction allows chemists and chemical engineers to peer into epidemic models. Moreover, the analogy illustrates how concepts in one field can transfer, aid understanding, and generate insights to/in another field.

\section{References}

[1] Fred Brauer. Mathematical epidemiology: Past, present, and future. Infectious Disease Modelling, 2(2):113-127, 2017.

[2] Herbert W Hethcote. The mathematics of infectious diseases. SIAM Review, 42(4):599-653, 2000.

[3] Fred Brauer. Compartmental models in epidemiology. In Mathematical Epidemiology, pages 19-79. Springer, 2008.

[4] William Ogilvy Kermack and Anderson G McKendrick. A contribution to the mathematical theory of epidemics. Proceedings of the Royal Society A: Mathematical, Physical and Engineering Sciences, 115(772):700-721, 1927.

[5] Roy M Anderson. Discussion: the kermack-mckendrick epidemic threshold theorem. Bulletin of Mathematical Biology, 53(1-2):1, 1991.

[6] James D Murray. Epidemic models and the dynamics of infectious diseases. In Mathematical Biology, pages 610-650. Springer, 1993.

[7] Leah Edelstein-Keshet. Mathematical Models in Biology. SIAM, 1988.

[8] Peter Schuster. What is special about autocatalysis? Monatshefte für Chemie-Chemical Monthly, pages 1-13, 2019.

[9] SK Scott. Reversible autocatalytic reactions in an isothermal CSTR: multiplicity, stability and relaxation times. Chemical Engineering Science, 38(10):1701-1708, 1983.

[10] James C Frauenthal. Mathematical modeling in epidemiology. Springer Science \& Business Media, 2012.

[11] Maia Martcheva. An Introduction to Mathematical Epidemiology, volume 61. Springer, 2015. 
[12] Edwin B Wilson and Jane Worcester. The law of mass action in epidemiology. Proceedings of the National Academy of Sciences, 31(1):24, 1945.

[13] Howard Howie Weiss. The SIR model and the foundations of public health. MATerials MATemátics, pages 0001-17, 2013.

[14] Matt J Keeling and Pejman Rohani. Modeling infectious diseases in humans and animals. Princeton University Press, 2011.

[15] Paul EM Fine. The interval between successive cases of an infectious disease. American Journal of Epidemiology, 158(11):1039-1047, 2003.

[16] H Scott Fogler. Essentials of Chemical Reaction Engineering. Pearson Education, 2010.

[17] Paul L Delamater, Erica J Street, Timothy F Leslie, Y Tony Yang, and Kathryn H Jacobsen. Complexity of the basic reproduction number $\left(R_{0}\right)$. Emerging Infectious Diseases, 25(1):1, 2019.

[18] Ottar N Bjornstad, S Katriona, and Martin Krzywinski. Modeling infectious epidemics. Nature Methods, 17:455-456, 2020.

[19] Tunde Tajudeen Yusuf and Francis Benyah. Optimal control of vaccination and treatment for an SIR epidemiological model. World Journal of Modelling and Simulation, 8(3):194-204, 2012.

[20] Boris Shulgin, Lewi Stone, and Zvia Agur. Pulse vaccination strategy in the SIR epidemic model. Bulletin of Mathematical Biology, 6o(6):1123-1148, 1998.

[21] Linda JS Allen. A primer on stochastic epidemic models: Formulation, numerical simulation, and analysis. Infectious Disease Modelling, 2(2):128-142, 2017.

[22] Abba B Gumel, Shigui Ruan, Troy Day, James Watmough, Fred Brauer, P Van den Driessche, Dave Gabrielson, Chris Bowman, Murray E Alexander, Sten Ardal, et al. Modelling strategies for controlling sars outbreaks. Proceedings of the Royal Society of London. Series B: Biological Sciences, 271(1554):2223-2232, 2004.

[23] Maia Martcheva. Techniques for computing $\mathcal{R}_{0}$. In An Introduction to Mathematical Epidemiology, pages 91-121. Springer, 2015.

[24] Ana Luz Vivas-Barber, Carlos Castillo-Chavez, and Ernest Barany. Dynamics of an SAIQR Influenza Model. Biomath, 3(2):1409251, 2014.

[25] Pauline van den Driessche. Reproduction numbers of infectious disease models. Infectious Disease Modelling, 2(3):288-303, 2017.

[26] P Van den Driessche and James Watmough. Further notes on the basic reproduction number. In Mathematical Epidemiology, pages 159-178. Springer, 2008.

[27] Odo Diekmann, Johan Andre Peter Heesterbeek, and Johan AJ Metz. On the definition and the computation of the basic reproduction ratio $R_{0}$ in models for infectious diseases in heterogeneous populations. Journal of Mathematical Biology, 28(4):365-382, 1990. 
[28] Eli P Fenichel, Carlos Castillo-Chavez, M Graziano Ceddia, Gerardo Chowell, Paula A Gonzalez Parra, Graham J Hickling, Garth Holloway, Richard Horan, Benjamin Morin, Charles Perrings, et al. Adaptive human behavior in epidemiological models. Proceedings of the National Academy of Sciences, 108(15):6306-6311, 2011.

[29] Timothy C Reluga. Game theory of social distancing in response to an epidemic. PLoS Computational Biology, 6(5), 2010.

[30] David N Fisman. Seasonality of infectious diseases. Annual Review of Public Health, 28:127-143, 2007.

[31] Joan L Aron and Ira B Schwartz. Seasonality and period-doubling bifurcations in an epidemic model. Journal of Theoretical Biology, 110(4):665-679, 1984.

[32] Ottar N Bjornstad, S Katriona, and Martin Krzywinski. The seirs model for infectious disease dynamics. Nature Methods, 17:557-558, 2020.

[33] David JD Earn. A light introduction to modelling recurrent epidemics. In Mathematical Epidemiology, pages 3-17. Springer, 2008.

[34] Linda JS Allen. An introduction to stochastic epidemic models. In Mathematical epidemiology, pages 81-130. Springer, 2008.

[35] Tom Britton. Stochastic epidemic models: a survey. Mathematical Biosciences, 225(1):24-35, 2010.

[36] Radek Erban, Jonathan Chapman, and Philip Maini. A practical guide to stochastic simulations of reaction-diffusion processes. arXiv preprint arXiv:0704.1908, 2007.

[37] Daniel T Gillespie, Andreas Hellander, and Linda R Petzold. Perspective: Stochastic algorithms for chemical kinetics. The Journal of Chemical Physics, 138(17):05B201_1, 2013.

[38] Herbert W Hethcote and David W Tudor. Integral equation models for endemic infectious diseases. Journal of Mathematical Biology, 9(1):37-47, 1980.

[39] Alun L Lloyd. Destabilization of epidemic models with the inclusion of realistic distributions of infectious periods. Proceedings of the Royal Society of London. Series B: Biological Sciences, 268(1470):985-993, 2001.

[40] P Van den Driessche. Spatial structure: Patch models. In Mathematical epidemiology, pages 179-189. Springer, 2008.

[41] Julien Arino, Jonathan R Davis, David Hartley, Richard Jordan, Joy M Miller, and P Van Den Driessche. A multi-species epidemic model with spatial dynamics. Mathematical Medicine and Biology, 22(2):129-142, 2005.

[42] Duygu Balcan, Bruno Gonçalves, Hao Hu, José J Ramasco, Vittoria Colizza, and Alessandro Vespignani. Modeling the spatial spread of infectious diseases: The global epidemic and mobility computational model. Journal of Computational Science, 1(3):132-145, 2010. 
[43] Stephen Eubank, Hasan Guclu, VS Anil Kumar, Madhav V Marathe, Aravind Srinivasan, Zoltan Toroczkai, and Nan Wang. Modelling disease outbreaks in realistic urban social networks. Nature, 429(6988):180-184, 2004.

[44] István Z Kiss, Joel C Miller, Péter L Simon, et al. Mathematics of epidemics on networks. Springer, 598, 2017.

[45] Shweta Bansal, Bryan T Grenfell, and Lauren Ancel Meyers. When individual behaviour matters: homogeneous and network models in epidemiology. Journal of the Royal Society Interface, 4(16):879-891, 2007.

[46] Matt J Keeling and Ken TD Eames. Networks and epidemic models. Journal of the Royal Society Interface, 2(4):295-307, 2005.

[47] Mark EJ Newman. Spread of epidemic disease on networks. Physical Review E, 66(1):016128, 2002.

[48] James O Lloyd-Smith, Sebastian J Schreiber, P Ekkehard Kopp, and Wayne M Getz. Superspreading and the effect of individual variation on disease emergence. Nature, 438(7066):355-359, 2005.

[49] Lauren Ancel Meyers, Babak Pourbohloul, Mark EJ Newman, Danuta M Skowronski, and Robert C Brunham. Network theory and sars: predicting outbreak diversity. Journal of Theoretical Biology, 232(1):71-81, 2005.

[50] Antoine Allard, Cristopher Moore, Samuel V Scarpino, Benjamin M Althouse, and Laurent Hébert-Dufresne. The role of directionality, heterogeneity and correlations in epidemic risk and spread. arXiv preprint arXiv:2005.11283, 2020.

[51] Maia Martcheva. Vector-borne diseases. In An Introduction to Mathematical Epidemiology, pages 67-89. Springer, 2015.

[52] David L Smith, Katherine E Battle, Simon I Hay, Christopher M Barker, Thomas W Scott, and F Ellis McKenzie. Ross, macdonald, and a theory for the dynamics and control of mosquito-transmitted pathogens. PLoS Pathogens, 8(4), 2012.

[53] Christopher Rackauckas and Qing Nie. Differentialequations.jl a performant and feature-rich ecosystem for solving differential equations in Julia. Journal of Open Research Software, 5(1), 2017. 


\section{A1 the appendix}

\section{A1.1 simulating the SIR model in Julia}

Code in the Julia language to numerically approximate the solution to the SIR model, via the DifferentialEquations.jl [53] package, is below.

using DifferentialEquations

RO $=2.0 \quad \#$ basic reproduction number

\# right-hand side of the ODE, viewed as:

\# $d u / d t=f=f(u, p, t)$

\# where u $:=[S, I, R]$

function update_f!( $f, u, p, t)$

\# for clarity, unpack vector u

$\mathrm{s}=\mathrm{u}[1]$

$i=u[2]$

$r=u[3]$

\# update $f$

$f[1]=-R 0 * s * i$

$\mathrm{f}[2]=\mathrm{R} 0 * \mathrm{~s} * \mathrm{i}-\mathrm{i}$

$f[3]=i$

end

\# initial condition

eps $=10^{\wedge}-5$ \# initial fraction infectious

$\mathrm{u} 0=[1-$ eps; eps; 0.0$]$

\# define the ODE problem

time_span $=(0.0,25.0)$

prob $=$ ODEProblem(update_f!, u0, time_span)

\# numerically solve ODE

sol $=$ solve (prob)

\# obtain numerical approximation to the solution at e.g. $t=10$

$\operatorname{sol}(10.0)$

A1.2 an alternative visualization of SIR model dynamics

Fig. A7 presents an alternative visualization of SIR model dynamics that emphasizes $[S](t)+[\mathrm{I}](t)+$ $[R](t)=1$ for all $t>0$. 


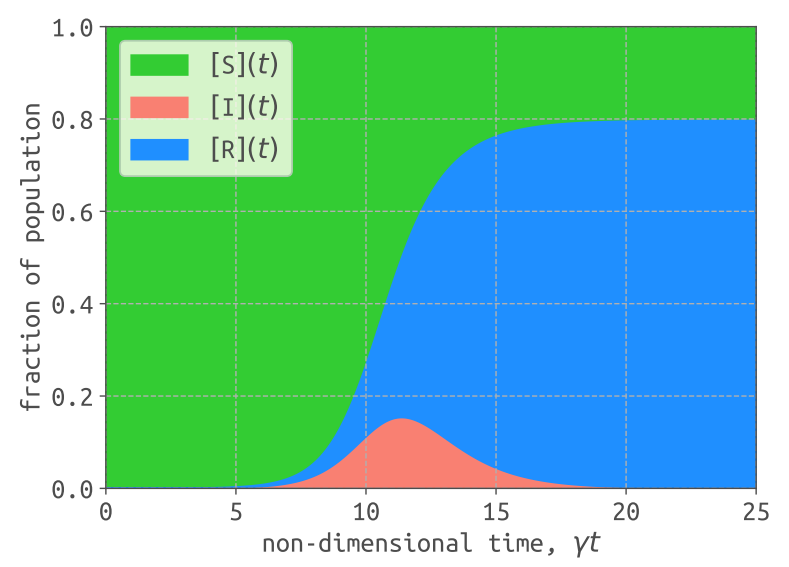

Figure A7: Numerical approximation of the solution to the SIR model in eqns. 1 1 3 for $\mathcal{R}_{0}=2$ and initial conditions in eqns $68_{0}\left([]_{0}=1 e-5\right)$. At any given time $t$, the proportion of the panel colored green, red, and blue, respectively, represents the fraction of the population in the susceptible, infectious, and recovered compartment.

\section{A1.3 early exponential growth of [I]}

See Fig. A8 to see how the approximation of $[I](t)$ in eqn. 9 fares.

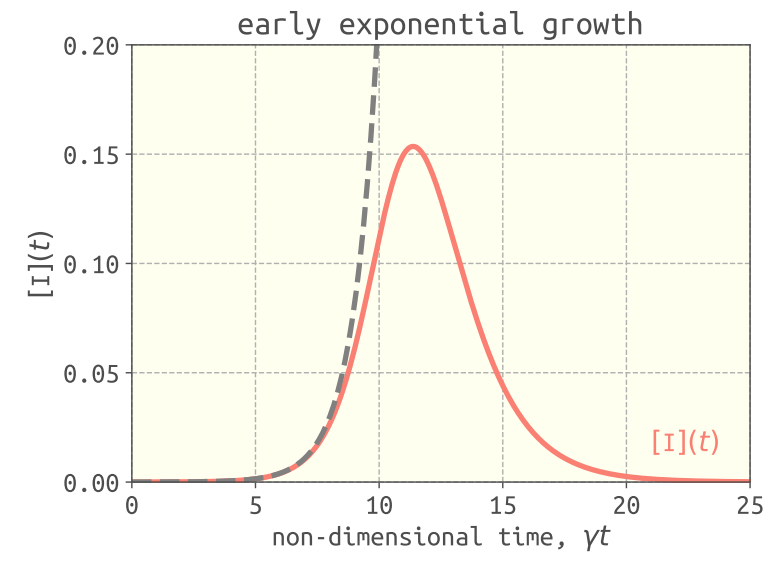

(a)

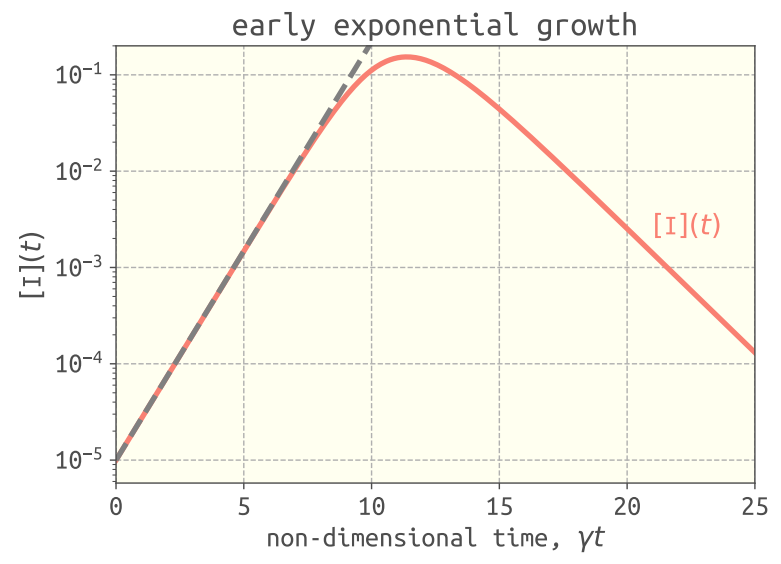

(b)

Figure A8: The $[I](t)$ curve from Fig. 2along with eqn.9 (gray, dashed line) to show that $[I](t)$ exhibits, approximately, exponential growth in the early stage of the epidemic. The fraction of the population that is infectious, $[1](t)$, is shown on both a (a) linear and (b) log scale. 